\title{
EDITORIAL
}

\section{Open surgery for thoracic aortic disease}

\section{H J Safi, P R Taylor}

While new technologies appear to offer potential advantages over traditional therapies for thoracic aortic disease, open surgery is still the mainstay of treatment for the overwhelming majority of patients

$M$ any recent technical advances have enhanced the safety of open surgery of the descending thoracic aorta. Previous to the refinement of these adjuncts and techniques, surgeons such as Stanley Crawford showed that simple aortic cross-clamping with expeditious surgery produced the best results. ${ }^{1}$ In Crawford's era of "clamp and go", time limitations pressured surgeons to perform anastomoses rapidly with perfect haemostasis. The duration of aortic crossclamping was directly related to survival and to serious complications such as paraplegia and visceral ischaemia. The role of bypass, intercostal reimplantation, and cerebrospinal fluid drainage was unclear, in that none of these techniques appeared to be beneficial.

Cross-clamping the aorta below the left common carotid artery and above the coeliac axis increases proximal systemic pressure, which in turn increases the cerebrospinal fluid pressure. In addition, the mean arterial pressure distal to the clamp will fall, and therefore the distal spinal cord will be at risk of ischaemia from a combination of decreased arterial perfusion and increased cerebrospinal fluid pressure. Logically, techniques that help to reduce the cerebrospinal fluid pressure and increase the distal arterial pressure will help to treat these two adverse factors, and will consequently lower the incidence of paraplegia.

Cerebrospinal fluid drainage prevents elevation of the cerebrospinal fluid pressure, and several techniques can increase the distal arterial perfusion pressure, such as a simple shunt, partial heart bypass (from the left atrium or the pulmonary veins to the left common femoral artery) or full cardiopulmonary bypass. ${ }^{2}$ Currently, there is overwhelming evidence that keeping the cerebrospinal fluid pressure low is beneficial. Practitioners recommend that cerebrospinal fluid drainage be implemented whenever the aorta is clamped above the level of the coeliac axis. ${ }^{2}$

Arterial bypass techniques to perfuse the distal See end of article for authors' affiliations

.............................

Correspondence to: Mr Peter Taylor Department of General and Vascular Surgery, Guy's \& St Thomas' Hospital, Lambeth Palace Road London SE 1 7EH, UK; taylorvasc@aol.com anastomoses are performed. However, when the aorta bearing the coeliac axis, superior mesenteric artery, and both renal arteries is clamped and opened, none of these vital organs will be perfused. In this instance selective perfusion of the visceral arteries using an octopus system can minimise organ ischaemia. ${ }^{34}$ Current studies attach an additional benefit to cooling the kidneys although the advantage to cooling the remainder of the viscera is somewhat controversial.

The routine reimplantation of intercostals has further decreased the incidence of paraplegia. ${ }^{5}$ Some authorities use somatosensory evoked potentials or motor evoked potentials to identify the critical vessels for reimplantation, ${ }^{56}$ but the general consensus is that the large intercostals in the region of T8-T12 must be reperfused. Aneurysms that involve the descending and abdominal aorta used to be exposed by completely transecting the diaphragm. ${ }^{7}$ However, this is not necessary in the majority of cases, and if avoided, patients have better respiratory function and recover more quickly. ${ }^{7}$ Additional gains have been achieved with the use of moderate hypothermia, in which patient temperature is permitted to drift downward to between $32-33^{\circ} \mathrm{C}$.

\section{INDICATIONS FOR SURGERY IN DISSECTION AND ANEURYSMS}

Acute dissection is the most common catastrophe to affect the aorta, occurring more frequently than rupture of the abdominal aorta. Dissection of the ascending aorta, with or without involvement of the descending thoracic aorta, is known as Stanford type A. Type B involves the descending thoracic aorta. Aortic dissection is labelled acute when the clinical diagnosis is made within two weeks following the onset of symptoms, and chronic after two weeks. Surgery immediately follows patient stabilisation for acute type A dissection because of the poor outcome associated with delayed treatment.

The peak incidence of type $\mathrm{B}$ aortic dissection is in the seventh decade. Within 2-5 years following detection, $20-40 \%$ of type B dissections become aneurysmal. ${ }^{8}$ The lifetime probability of rupture in descending thoracic and thoracoabdominal aortic aneurysm is $75-80 \%$, with a five year untreated survival rate in the range of $10-20 \%{ }^{8}$ Identified risk factors for rupture include increasing aortic size, advanced age, raised mean arterial blood pressure, and associated chronic obstructive pulmonary disease.

The main rationale behind surgical graft replacement of descending thoracic and thoracoabdominal aorta is the prevention of fatal aortic rupture. Initially, because of the high rates of morbidity and mortality associated with surgical segment of aorta is clamped and opened.

Distal bypass assures good perfusion of the legs, viscera, and kidneys while the proximal 
intervention for type B dissection, patients are treated medically. This is primarily with the use of aggressive antihypertensive medication and analgesia. Surgical intervention is reserved for emergency patients threatened by aortic rupture, ischaemic complications, uncontrollable hypertension, or intractable pain.

The 30 day mortality rate for surgery delayed until the chronic stage is $7-14 \%$, with no greater risk than that for aneurysm graft replacement of the descending thoracic or thoracoabdominal aorta. ${ }^{8}$ Chronic type B aortic dissection was previously considered a risk factor for neurological complications, particularly during the "clamp and go" era. However, in 854 patients operated on for descending thoracic and thoracoabdominal aortic aneurysms, we found no difference in neurological outcome between patients with or without chronic dissection. Risk factors for neurological complications include prolonged aortic clamp time, extensive (type II) thoracoabdominal aortic aneurysms, and renal failure. The adjunctive techniques of distal aortic perfusion and cerebrospinal fluid drainage have reduced the overall incidence of neurological deficits to $0.9 \%$ for descending thoracic aortic aneurysm repair and to $3.3 \%$ for thoracoabdominal aortic aneurysm repair. ${ }^{9}{ }^{10}$ The most troublesome group of patients is still type II thoracoabdominal aortic aneurysms, with the highest rate of neurological complications. However, the beneficial effect of adjuncts is also most apparent in this group, having reduced the rate of paraplegia to approximately $7 \%$ from a former high of $33 \%$.

\section{CURRENT STATUS OF ENDOLUMINAL TREATMENT}

New technology is always attractive when it is associated with potential advantages over current treatment. Stent grafts have been used to treat pathology of the descending thoracic aorta. Avoiding thoracotomy, full heparinisation, and aortic crossclamping is beneficial. Reducing distal ischaemia to a minimum should decrease the incidence of paraplegia and visceral and renal ischaemia. However, the initial period of enthusiasm has been tempered with the publication of complications associated with these devices. These include rupture of the aorta caused by gross oversizing of the graft and fatal consequences associated with covering the coeliac axis. The number of neurological complications associated with endoluminal thoracic repair is increasing steadily, particularly paraplegia and stroke. Experience with stent grafts in the infrarenal aorta has shown that there is a risk of rupture of $1 \%$ per annum. The stent graft may become displaced and migrate, allowing the sac to become pressurised and therefore at risk of rupture. Several stent grafts have had to be withdrawn to be redesigned, often because of fractures of the stent or hooks. The definitive stent graft has not yet been manufactured, and although open surgery is associated with higher risks in the short term, the long term integrity of the grafts is not in doubt.

Randomised controlled trials offer the best way to compare open surgery and endoluminal repair. Although these are underway for infrarenal aortic aneurysms, no randomised trial has yet made this comparison for repair of the thoracic aorta. The use of registries may be a way forward to prove the efficacy of these devices outside the constraints of a randomised trial. However, until information is available on the long term durability for stent grafts placed in the thoracic aorta, open surgery for thoracic aortic pathology is still the mainstay of treatment for the overwhelming majority of patients

\section{Authors' affiliations}

H J Safi, Department of Cardiothoracic and Vascular Surgery, The University of Texas Health Science Center at Houston, Memorial Hermann Hospital, Houston, Texas, USA

P R Taylor, Department of General and Vascular Surgery, Guy's \& St

Thomas' Hospital, London, UK

\section{REFERENCES}

1 Crawford ES, Crawford JL, Safi HJ, et al. Thoracoabdominal aortic aneurysms: preoperative and intraoperative factors determining immediate and long-term results of operations in 605 patients. J Vasc Surg 1986;3:389-404

2 Safi HJ, Bartoli S, Hess KR, et al. Neurological deficit in patients at high risk with thoracoabdominal aortic aneurysms: the role of cerebral spinal fluid drainage and distal aortic perfusion. J Vasc Surg 1994:20:434-43.

3 Safi HJ, Miller CC 3rd, Yawn DH, et al. Impact of distal aortic and visceral perfusion on liver function during thoracoabdominal and descending thoracic aortic repair. J Vasc Surg 1998;27:145-53.

4 Jacobs MJ, Eijsman L, Meylaerts SA, et al. Reduced renal failure following thoracoabdominal aortic aneurysm repair by selective perfusion. Eur J Cardio Thor Surg 1998;14:201-5

5 Safi HJ, Miller CC 3rd, Carr C, et al. Importance of intercostal artery reattachment during thoracoabdominal aortic aneurysm repair. J Vasc Surg 1998;27:58-68.

6 Jacobs MJ, Meylaerts SA, De Haan P, et al. Strategies to prevent neurologic deficit based on motor-evoked potentials in type I and II thorocoabdominal aortic aneurysm repair. J Vasc Surg 1999;29:48-59.

7 Engle J, Safi HJ, Miller CC 3rd, et al. The impact of diaphragm management on prolonged ventilator support after thoracoabdominal aneurysm repair. J Vasc Surg 1999:29:150-6.

8 Huynh TT, Porat EE, Miller CC 3rd, et al. The effect of aortic dissection on outcome in descending thoracic and thoracoabdominal aortic aneurysm repair. Semin Vasc Surg 2002;15:108-15.

9 Estrera AL, Miller CC 3rd, Huynh TT, et al. Neurologic outcome after thoracic and thoracoabdominal aortic repair. Ann Thorac Surg 2001;72:1225-30.

10 Estrera AL, Rubenstein FS, Miller CC 3rd, et al. Descending thoracic aortic aneurysm: surgical approach and treatment using the adjuncts cerebrospinal fluid drainage and distal aortic perfusion. Ann Thorac Surg 2001;72:481-6. 